\title{
Dos psicólogos del siglo XX: Viktor E. Frankl y Hans Jürgen Eysenck, a dos décadas de su muerte
}

Dr. Ramón León Fernández

Hace veinte años fallecieron Viktor E. Frankl y Hans Jürgen Eysenck. En la presente comunicación el autor trata del significado de sus trabajos e ideas para la psicología. Frankl fue el fundador de la logoterapia, un enfoque que hoy día está difundido por todo el mundo. Eysenck sentó las bases de la terapia de la conducta y fue un importante investigador de la psicología de la personalidad.

Palabras clave: Viktor E. Frankl - Hans Jürgen Eysenck

\section{Two psychologists of the 20th century: Viktor E. Frankl and Hans Jürgen Eysenck, to two decades of their death}

Twenty years ago Viktor E. Frankl and Hans Jürgen Eysenck passed away. In the present communication the author discusses the significance of their works and ideas to psychology. Frankl was the founder of logotherapy, an important psychotherapy theory which at the present enjoys of worldwide attention, Eysenck put the basis for the behavioral therapy and was a very important researcher in the the psychology of personality.

Key words: Viktor E. Frankl - Hans Jürgen Eysenck 
Hace veinte años, a comienzos de setiembre de 1997, en el breve lapso de una semana, la psicología perdió a dos de sus más distinguidos exponentes: Viktor E. Frankl (fallecido el 2 de ese mes) y Hans Jürgen Eysenck (fallecido el 4). La noticia tomó de sorpresa a muchos, que a pesar de la edad avanzada de ambos (en especial de Frankl), los imaginaban (y los deseaban) muy lejanos todavía de la muerte: tal la vitalidad de su acción, tal el significado de sus ideas para psiquiatras, psicoterapeutas y psicólogos.

Ambos ocupaban ya en vida un lugar destacado en la historia de la psicología: Eysenck era uno de los psicólogos más citados (Rushton 2001) y Frankl el creador de un sistema de psicoterapia de difusión mundial.

Las razones para el reconocimiento de uno y otro son de distinta índole, pues sus contribuciones son de diferente orientación y distinta naturaleza. Pero la eminencia de ambos, así como la excelencia y la influencia de su obra son indiscutibles.

Frankl enriqueció con su concepción de la logoterapia el arsenal psicoterapéutico, proponiendo un conjunto de ideas que buscan ofrecer al ser humano la posibilidad de responder afirmativa y originalmente a la pregunta cada vez más acuciante acerca del sentido de su existencia.

Eysenck, por su parte, defendió con laboriosidad incansable y convicción férrea la necesidad de una psicología basada en hechos, muchas veces fríos y hasta decepcionantes, pero siempre preferibles a las ilusiones, las preconcepciones y las especulaciones sin freno que -hay que reconocerlo- son moneda de difundida circulación en la psicología, la psiquiatría y la psicoterapia.

Tratar de Frankl y Eysenck, propósito de estas líneas escritas al cumplirse veinte años de la desaparición física de ambos, es referirse a dos de las mentes más creativas de la psicología del siglo XX, cuyas ideas, planteamientos y hallazgos (no siempre exentos de aspectos polémicos) aún hoy conservan gran importancia.

En sus carreras y en sus aportes, uno y otro resumen en buena medida la evolución de la ciencia psicológica en lo que Hobsbawm (1995) ha calificado como el siglo más corto de la historia.

En esa evolución, aun el más desavisado de los psicólogos reconocerá una escisión. De un lado, la insistencia de unos en conservar (y hasta reforzar) los estrechos vínculos de la psicología y la filosofía, de larga data además; del otro, el deseo de quienes aspiran y trabajan por una psicología con carta de ciudadanía científica, concentrada en la búsqueda de hechos y alejada de la especulación.

Frankl y Eysenck se ubican en una y otra posición. El primero, con su insistencia en la importancia del espíritu y, finalmente, de la subjetividad. El segundo, con la búsqueda decidida de la objetividad.

En la aventura intelectual que cada uno de ellos emprendió hay mucho de grandioso pero también de trágico. Acercarse a ellas, estudiarlas ofrece posibilidades de acceder a una visión diferenciada de la psicología y sus vicisitudes en la centuria pasada. 
Teóricos de talla, escritores con producción considerable, líderes de enfoques originales, defensores y difusores convencidos de sus respectivas posturas en el mundo de la psicología, Frankl y Eysenck vivieron una vida marcada por los dramáticos sucesos de la Europa de los años treinta y cuarenta pero, tras los tiempos de dolor, destrucción e incertidumbre, supieron trabajar con tenacidad para encumbrarse en la psicología, y, al hacerlo, han dejado un legado de inestimable valor.

\section{Viktor E. Frankl}

Viktor E. Frankl nació en 1905 en Viena, entonces todavía la capital del Imperio Austro-Húngaro. La imagen romántica de esa metrópoli (asociada al Danubio Azul, de Johann Strauss), sin embargo, no corresponde a la realidad del día a día que con toda seguridad pudo percibir Frankl en sus años de niñez y adolescencia.

En Viena infame y genial, una historia de esa ciudad de fines del siglo XIX y comienzos del XX, Riedl (1995) señala que "si Viena es una metrópoli, lo es de la envidia y la indiferencia" (pg. 29), agregando que hacia 1920, "hacía mucho que la pequeña burguesía, bajo el alcalde socialcristiano antisemita Karl Lueger (un desertor de los liberales), se había hecho con el poder" y que, asimismo, "hacía mucho que el exhausto proletariado no aguantaba en los barrios miserables de los distritos obreros" (pg. 57). Pero no solo Riedl tiene una opinión tan sombría de esa urbe. También la tiene Casals (2015): "Viena es a la vez la capital de la verdad y la simulación. Es decir de la ambivalencia" (pg. 33).

Una atmósfera sombría se cernía sobre el Imperio Austro-Húngaro. Francisco José, el emperador que regiría el destino de ese conglomerado de pueblos entre 1848 y 1916, era, por cierto, para entonces, el gobernante europeo de más experiencia, respetado por propios y extraños (Kagan 2003). A pesar de su experiencia y del respeto que él inspiraba, Francisco José no podía impedir que el Imperio Austro-Húngaro se encaminara en el indetenible proceso de su decadencia: más allá de su importancia para la estabilidad política de la Europa de aquel entonces, los signos de su descomposición política y social se manifestaban con claridad.

Casals (2015) habla de un aura de irrealidad que envolvía a esa nación y que nadie mejor que el propio emperador encarnaba:

"a la vez que mantiene buenas relaciones con la burguesía judía -base de apoyo del liberalismo-, Francisco José sigue aferrado en la vida de la corte a un ceremonial barrocoabsolutista, se hace llamar Seine Apostolische Majestät unser allergnädigster Kaiser und Herr, preserva cuidadosamente los lazos con la púrpura y con las ochenta grandes familias, y muestra una particular afección por los uniformes y los aspectos decorativos de la milicia" (pg. 31).

En esa atmósfera transcurrió la niñez de Frankl. La preocupación por los temas filosóficos y antropológicos fue notoria en él desde muy temprano. Lukas (1985) anota que ya a los 16 años expuso sobre el sentido de la vida en una escuela popular, y que los estudios de secundaria los concluyó con un trabajo (Maturaarbeit) que llevaba por título "Zur Psychologie des philosophischen Denkens" [Acerca de la psicología del pensamiento filosófico]. 
Es también en estos años de adolescencia que se siente atraído por el psicoanálisis. En su autobiografía (Frankl 1995) recuerda sus tempranos contactos epistolares con Sigmund Freud (1856-1939), mencionando con orgullo la decisión de éste de publicar un trabajo suyo en la Internationale Zeitschrift für Psychoanalyse, la revista oficial del movimiento psicoanalítico.

A pesar de este auspicioso acontecimiento, Frankl no se adhirió al psicoanálisis. El paso del tiempo lo llevó a acercarse, más bien, en 1924 a Alfred Adler (1870-1937, el creador de la Psicología Individual), primero discípulo y después disidente de Freud.

La concepción escéptica, casi trágica del ser humano, inherente al pensamiento freudiano, así como la insistencia del padre del psicoanálisis en el poder omnímodo del instinto, fueron probablemente la causa de su alejamiento de Freud. Adler, con una perspectiva más optimista acerca de las posibilidades del hombre y con su cercanía al discurrir de la vida, ejerció durante una época gran atracción sobre Frankl. El encuentro entre ambos, lo recuerda Frankl (1995), se produjo por mediación de Hugo Lukacs, en el Café Siller, donde el padre de la Individualpsychologie se reunía con sus discípulos:

"Éste [Adler; R.L.] aceptó sin mayores dudas el manuscrito de mi artículo "Psychotherapie und Weltanschauung", el cual sorprendentemente apareció en su Internationale Zeitschrift für Individualpsychologie, tan solo un año después de mi publicación en la revista psicoanalítica".

En 1926, con solo 21 años y aun en el curso de sus estudios de medicina, Frankl presentó una ponencia en el Tercer Congreso Internacional de Psicología Individual, en Düsseldorf (Danzer 2002).

Con el paso del tiempo, sin embargo, también habría de tomar distancia de Adler. Este, que no había tolerado el autoritarismo de Freud, ejercía un control no menos estricto sobre sus seguidores, exigiéndoles la plena, total aceptación de sus ideas, razón por la cual separó de su entorno a Rudolf Allers (1883-1963) y Oswald Schwarz (1883-1949) cuando éstos le plantearon algunas observaciones críticas. Como Frankl se solidarizara con ellos, en especial con el primero, Adler forzó también su separación. En 1927 Frankl se alejó definitivamente del círculo adleriano.

De allí en adelante, Frankl, médico aún sumamente joven, desarrolló su carrera profesional en Viena, la misma que se vio interrumpida con la invasión de Austria por parte de las tropas nacionalsocialistas en 1938. Pero ya en ese año, da a la publicidad un artículo en el Zentralblatt für Psychotherapie con el título de "Zur geistigen Problematik der Psychotherapie" (Frankl 1938).

Los violentos cambios sociales que se produjeron a partir de entonces afectaron de modo dramático a Frankl, quien habría de vivir la experiencia del campo de concentración, en el que perdió a sus padres, su hermano y su primera esposa, Tilly.

La vida en el campo de concentración, con sus indecibles humillaciones cotidianas, con sus maltratos y la consecuente merma de la dignidad humana, constituía una experiencia de suma crueldad, de odio insondable, que derrotó a la mayoría de los prisioneros mucho antes que perecieran en las cámaras de gas. El absurdo, el rotundo sinsentido de todo lo que allí ocurría, el odio inmisericorde y la degradación sistemática de la condición humana, solo podían causar desesperación o, en caso contrario, una apatía que era el anticipo mismo de la desaparición física. 
Frankl sobrevivió a todo esto. Lo hizo, señala él, gracias a una actitud vital en la cual cada nuevo día era visto por él como un regalo divino, con tareas específicas a cumplir a fin de demostrarse a sí mismo que conservaba la vida y la autonomía espiritual mínima para dar sentido a su existencia, con el propósito de no dejarse ganar por la desesperación, el odio o la resignación.

Cuando la Segunda Guerra Mundial finalizó, llegó el momento para el despliegue de sus potencialidades. El conjunto de ideas que paulatinamente elaboró y que constituye su sistema psicoterapéutico, la logoterapia, tiene como eje central la experiencia y la vivencia del sentido y el vacío existenciales.

Pocos estaban tan familiarizados con el sinsentido, el vacío existencial y hasta el absurdo como Frankl. En su adolescencia fue testigo de la Primera Guerra Mundial y asistió al traumático derrumbe del Imperio de los Habsburgo y la grave crisis social que vino después. Su mundo de todos los días fue sepultado, arrasado por la vorágine de los acontecimientos, de modo tal que se transformó en el mundo de ayer, para emplear las palabras que utilizara Stefan Zweig en el libro nostálgico que lleva ese título (Zweig 2001).

En claro deslinde con Freud, Frankl considera que el problema central del hombre en el siglo XX es la búsqueda de sentido para su existencia. No es la sexualidad la que le causa angustia, sino más bien el no hallar un sentido a muchas de las cosas que ocurren en su vida.

No siempre es fácil expresar ideas sobre la existencia, su sentido, sus fines y sus dilemas y encrucijadas. Filósofos y metafísicos, angustiados por esa problemática y anhelantes de descifrar su esencia, han tomado la pluma y nos han dejado obras en las cuales compiten la angustia y la esperanza, la voluntad afirmativa en unos casos y en otros la visión nihilista. Y al hacerlo muchas veces han plasmado sus ideas en un modo que angustia no tanto por el mensaje como más bien por la obscuridad, por lo idiosincrásico del estilo empleado.

El caso más dramático lo constituye, sin duda, Heidegger (1889-1976), cuyo estilo rebuscado, ambiguo cuando no oscuro, rico en neologismos, proclive a un desconcertante juego de palabras y por momentos con ciertos visos poéticos, raya con frecuencia en lo incomprensible. No fue ese el caso de Frankl: su producción escrita, pertinaz, le permitió difundir rápidamente sus ideas, gracias a un estilo que llega al lector y que se pone de manifiesto en artículos, libros, conferencias, clases y transmisiones radiales (Danzer 2002).

\section{Esencia y técnicas de la logoterapia}

La logoterapia, aunque sensu stricto una terapia, es en realidad una forma de ayuda, de búsqueda del camino a la madurez y al desarrollo existencial, también a la disposición de quien, libre de síntomas y de trastornos, siente que algo esencial falta en su vida; que hay espacios y dimensiones en su existencia que le son ajenos, a los cuales él no puede acceder.

\section{Ya lo dice Pongratz (1973):}

"Esta terapia no es apropiada ciertamente para cualquiera -ni para cualquier paciente ni para cualquier terapeuta. ¿Cuándo está indicada? Frankl menciona tres condiciones: el que busca 
ayuda debe de manera espontánea evidenciar sus necesidades espirituales; debe ser por lo general "una persona de tipo intelectual". Se da por sentado además la sensibilidad interna frente a los problemas acerca de la concepción de la vida y del mundo y la capacidad para tomar posición con respecto a ellos. Por último, la logoterapia es el método de elección cuando se trata de elaborar y aceptar golpes del destino (invalidez, enfermedades incurables). De este último criterio de indicación se deduce además que la logoterapia no solo es una terapia de la neurosis. Ella es también ayuda en crisis y en el proceso de maduración" (pp. 254-255).

No debemos olvidar, empero, que Frankl era médico y que, siguiendo su vocación de tal, tenía como primer objetivo curar, ayudar, aliviar, ofrecer apoyo o quizás -en el peor de los casos- solo consuelo a los que sufren.

Dejemos, por ello, que él mismo nos presente de modo resumido la esencia de la logoterapia:

"Concebida específicamente como psicoanálisis, la psicoterapia tiende hacia un resultado que es, concretamente, el de tornar consciente lo psíquico. La logoterapia, por el contrario, tiende a tornar consciente lo espiritual" (Frankl 1970; pg. 38).

Pongratz, ya citado, nos da elementos de juicio adicionales, que deseamos transcribir:

"La psicoterapia de Frankl debe ser entendida como una terapia "desde lo espiritual hacia lo espiritual". "Desde lo espiritual" significa que ella se dirige al ser humano como un ser espiritual, dando por sentado la presencia de elementos espirituales. "Hacia lo espiritual": que la terapia busca que actualizar esas posibilidades, allí donde están adormecidas o reprimidas" (pg. 254).

La logoterapia conjuga elementos propios del psicoanálisis, la antropología filosófica de Max Scheler (1874-1928), la psicología individual de Adler y la filosofía existencial. Danzer (2003) señala que Martín Heidegger, Karl Jaspers (1883-1969), Martin Buber (1878-1965) y Gabriel Marcel (18891973) eran referentes de significado para Frankl, y agrega que

"al lado de ellos Frankl menciona en sus escritos con frecuencia nombres y conceptos de Max Scheler, Erwin Straus, Ludwig Binswanger o Medard Boss y, por supuesto, los de Freud, Adler y Jung" (pg. 69).

Como consecuencia natural de su preocupación por el sentido de la vida, Frankl ingresa a la consideración del mundo de los valores. La clasificación que hace de éstos en estéticos, de actividad y de actitud, proviene de la teoría de la ética de Max Scheler, si bien Frankl agrega elementos de reflexión personal (Spiegelberg 1985). Fizzotti (1977) detalla la relación entre las ideas de Max Scheler y Viktor Frankl:

"Frankl mantiene, como Scheler, que el problema central y esencial es el de la existencia espiritual frente a la facticidad física. Cuando Frankl habla de 'antagonismo psiconoético' quiere resaltar la existencia espiritual de un ser libre y responsable contra el aparente condicionamiento a partir de la facticidad psicofísica. Además, si Scheler habla del hombre abierto al mundo, Frankl sostiene que ser hombre quiere decir ser más allá de sí mismo, en el sentido de que la esencia de la existencia humana consiste en la autotrascendencia" (pg. 85). 
Por su parte, la visión optimista y referida al futuro, a lo potencial, que está presente en la vida de todo individuo, nos recuerda a las ideas de Alfred Adler.

Ahora bien, en términos concretos, ¿a quiénes, de todos los que sufren se dirige la logoterapia?

Elizabeth Lukas (2015), tal vez la más profunda conocedora de la obra de Frankl (con quien tuvo trato directo y continuado por años), señala cinco campos de aplicación de la logoterapia: neurosis noogenas o existenciales (la midlife crisis, por ejemplo) y depresiones, neurosis psicógenas y enfermedades psicosomáticas, psicosis endógenas y enfermedades incurables, frustraciones existenciales y desorientación valorativa, y las psicopatologías propias de cada época.

El aporte conceptual de Frankl no está circunscrito solo al dominio de la doctrina, al mundo de las ideas. El conocido psicoterapeuta lo complementó con técnicas específicas y de inteligente propósito, como la intención paradójica y la derreflexión. La primera corresponde a la técnica de la "práctica negativa" (Dunlap 1928, 1932).

Constituye además la logoterapia la Tercera Escuela Vienesa de la Psicoterapia, después del psicoanálisis y la psicología individual.

\section{Viena y la logoterapia}

Tres grandes escuelas psicoterapéuticas desarrolladas en Viena: ¿casualidad? ¿hecho fortuito? No lo creemos. El singular ambiente de Viena, lugar de encuentro de varias culturas, antesala de Europa Oriental, sede de los Habsburgo, con sus grandezas y sus miserias, debe haber sido un poderoso catalizador para espíritus como el de Freud y el de Adler.

Frankl fue, por cierto, digno sucesor de ellos. Pero su relación con la gran ciudad fue menos conflictiva que la que tuvieron Freud y Adler. Recordemos los sentimientos ambivalentes del padre del psicoanálisis hacia Viena; y también cómo Adler progresivamente se alejó de ella y buscó horizontes más receptivos para sus ideas en el Nuevo Mundo.

No fue ese el caso de Frankl; en modo alguno él dio pábulo con sus ideas a las resistencias y el rechazo que Freud habría de despertar, ni fue menospreciado o sencillamente ignorado, como Adler. Muy por el contrario: a diferencia de uno y de otro, Frankl fue abrumado de honores y reconocimientos formales e informales, tanto en su ciudad como en el mundo entero. Solo para mencionar un ejemplo: Frankl fue Profesor de Neurología y Psiquiatría de la Universidad de Viena, distinción que no pudieron alcanzar ni Freud ni Adler.

Viena era para Frankl la ciudad de los mil recuerdos. Los de su infancia, los de sus años formativos, durante los cuales -como ya se dijo- Freud y Adler desempeñaron un rol de gran significado. Pero no solo ellos: también Rudolf Allers, el brillante crítico del psicoanálisis, y Otto Pötzl (1877-1962), el célebre sucesor del laureado Julius Wagner von Jauregg (1857-1940) en la Universidad de Viena.

A todos ellos Frankl ha rendido homenaje con sus escritos (Frankl 1994). Un homenaje que suponía una crítica en el caso de Freud y de Adler. Puede esto parecer paradójico, pero es que en el mundo de la ciencia sólo se hace acreedor a la crítica lo que es original y, aunque cuestionable, sugerente. 
Su obra, extensa y rica, ofrece al ser humano la posibilidad de una visión esperanzadora de su destino y del sentido de su vida. La modernidad y el mundo de hoy han transformado de modo dramático la vida, el modo de pensar y la forma de verse del hombre de hoy. Frankl fue consciente de eso: su obra es la respuesta suya al drama del hombre de nuestros días.

\section{La obra escrita de Frankl}

En modo alguno Frankl se limitó a la práctica privada, que alcanzó en su caso dimensiones importantes, ni a la tarea (muy agradable para él) de conferencista de nivel mundial. Al lado de esto, en alarde de energía casi inagotable, dedicó numerosas horas a la preparación de una obra escrita que es su legado a las generaciones venideras. Según el recuento de Lukas (2015), los 39 libros que escribió han sido traducidos a 40 idiomas.

Destacan en su obra escrita, como es lógico dedicada a la exposición y difusión de sus ideas, un par de títulos que no pueden obviarse al momento de hacer un recuento de su producción bibliográfica.

Nos referimos a Trotzdem ja zum Leben sagen (traducido al castellano como El hombre en busca de sentido, Frankl 1991), y Existencialismo y psicoanálisis (Frankl 1970). Volveremos a ambos un poco más adelante. Al lado de esos libros hay muchos más: ¿qué lector de habla castellana interesado en la psicoterapia no ha visto en los armarios de librerías y bibliotecas títulos como Teoría y terapia de las neurosis (Frankl 1964), o La presencia ignorada de Dios (Frankl 1979)?

Tal producción de libros y artículos no ha dejado de generar opiniones críticas negativas. Nada menos que en una nota necrológica aparecida en la Franfurter Allgemeine Zeitung, Weinzierl (1997) escribe:

"No solo el carácter accesible para todos de las tesis de Frankl fue la causa de su éxito: sus treinta libros fueron traducidos en más de veinte idiomas, solo en los Estados Unidos se vendió más de tres millones de ejemplares de Man's search for meaning. Por cierto, a veces recuerdan sus tratados menos a resultados de la investigación que al folletín filosófico, que quiere en primera línea ofrecer ayuda en la vida de las personas" (pg. 35).

Una crítica todavía más severa es la que propone Rattner (1990). Aunque reconoce sus cualidades literarias así como su perspicacia para reconocer los unilateralismos de otras doctrinas, para él Frankl es solo un acróbata intelectual:

"Un orgullo desmedido, inteligencia aguda y retórica que convence son las características de Frankl que le han permitido el éxito: es dudoso si ese éxito será duradero" (pg. 746).

El hombre en busca de sentido es todo un best seller. La obra es una exposición en verdad conmovedora de la vida en los campos de concentración. Se trata de un libro que describe una situación límite, a la cual Frankl logró sobrevivir. Se la puede leer como el testimonio de quien pudo salir indemne de una de las experiencias más brutales que la maldad humana ha podido imaginar y transformar en espantosa realidad. Pero, igualmente, es un libro que explora las inmensas posibilidades de la persona humana para enfrentar situaciones indecibles ${ }^{1}$. 
Existencialismo y psicoanálisis es, de otro lado, una exposición a la vez histórica y técnica de sus ideas, en la cual es posible reconocer con claridad las influencias de diferentes pensadores en Frankl, al mismo tiempo que la originalidad de muchos de sus planteamientos. En sus páginas Frankl desarrolló la clasificación de los valores a la que hemos hecho referencia líneas atrás.

\section{Hans Jürgen Eysenck}

Nacido en 1916 en Berlín, Hans Jürgen Eysenck tuvo desde muy joven una individualidad definida, que lo llevaría a rozamientos y fricciones con una sociedad como la alemana, que se dirigía a su época más negra.

Los años de su adolescencia coincidieron con los de la llegada al poder de Hitler. Negándose a admitir las normas de conducta impuestas por los nacionalsocialistas, Eysenck se ganó más de un problema, entre ellos el de su rechazo por parte de la Universidad de Berlín. La emigración, primero a Francia, y después a Inglaterra en 1934, terminaría alejándolo definitivamente de su patria.

Con interés por las ciencias naturales, Eysenck llegó a la psicología por casualidad, casi por descarte. En Francia estudiaría literatura e historia, en Dijón, lo mismo que hizo en Exeter, en Inglaterra. Después optaría por la psicología.

Los estudios de psicología Eysenck los realizaría en el University College de Londres, en el cual a diferencia de lo que ocurría en Cambridge, no se privilegiaba el trabajo experimental sino el estudio de las diferencias individuales, siguiendo la tradición de Galtron.

Cyril Burt (1883-1971), poseedor de una erudición impresionante así como de un gran conocimiento de la estadística, era la figura más destacada de la psicología británica, que vivía en ese entonces su época de oro: al lado de él, Godfrey Thomson (1881-1955) era el otro gran nombre de la época. En especial, Burt, bajo cuya dirección se formó Eysenck, representaba y continuaba de modo brillante la larga tradición matemática de la psicología inglesa, cuyas bases fueron sentadas por Francis Galton (1822-1911) y Charles Spearman (1863-1945).

Personalidad compleja, difícil, y tras unos modales exquisitos, de un gran autoritarismo, Burt solía despertar sentimientos ambivalentes; la relación de Eysenck con él no constituyó la excepción. La vinculación entre ambos, inicialmente la de un profesor con un alumno, devino conflictiva en lo personal, con intentos por parte de Burt de frenar la carrera de su aprovechado discípulo. Sería más bien el psiquiatra Aubrey Lewis (1900-1975)² quien la impulsaría, al invitar a Eysenck a integrarse al Mill Hill Hospital.

La inteligencia del joven estudiante, sus elevadas ambiciones, el rigor de su formación, se conjugaron en la actividad de Eysenck, plasmada en una impresionante bibliografía. Gudjonsson

\footnotetext{
1. Hasta donde conocemos, solo otro libro psicológico ha incidido en un tema como el abordado por Frankl en El hombre en busca de sentido. Nos estamos refiriendo a El corazón informado, de Bruno Bettelheim (1973).

2. Aubrey Lewis fue el primer Profesor del Instituto de Psiquiatría de la Universidad de Londres, entre 1946 y 1966, y tuvo un rol de primer orden en la psiquiatría británica de su época, especialmente en el desarrollo de la psiquiatría social (Sheperd 1980). Detenida información sobre su vida y su obra puede encontrarse en Goldberg et al. (2015).
} 
(1997) ha calculado en 79 libros y 1078 artículos el caudal de la su producción científica. Con solo 31 años, dio a la prensa una obra que tuvo gran resonancia: Dimensions of personality (Eysenck 1947).

Tal producción, portentosa en la cantidad, variada en la orientación y aclamada por unos así como cuestionada por otros, se caracteriza por algunos rasgos distintivos (Rushton 2001).

De un lado está la concentración en unos cuantos temas, en los que Eysenck ha hecho aportes o planteado sugerencias de significado: el estudio dimensional de la personalidad, el de la inteligencia, los aportes a la terapia del comportamiento. asi como una varia en la cual podemos encontrar hasta un singular libro sobre astrología (Eysenck \& Nias 1985).

Sus contribuciones al estudio de la personalidad y de la inteligencia son las que han tenido mayor difusión y repercusión en la psicología de hoy, de modo tal que, como anotan Costa \& McCrae (1986), aunque Eysenck es en el más amplio sentido del término un psicólogo general, en Estados Unidos se le conoce más como un teórico de la personalidad. Y, en los últimos años de su vida, su trabajo se orientó a la inteligencia, podríamos añadir.

Por otra parte, caracteriza a sus escritos el despliegue de técnicas matemáticas, que proviene de la larga tradición cuantificatoria de la psicología inglesa.

A no dudarlo, Eysenck poseía una de las más elegantes y expresivas plumas de la psicología contemporánea. Sus estudios de literatura, su vasta cultura lingüística, en general su inteligencia y su agudeza polémica, fueron responsables de esa cualidad que se revela de inmediato en sus obras que, sin embargo, poseen una seriedad que también se percibe muy rápidamente.

Una pluma excelente, una labor de investigación infatigable y claras (aunque no siempre aceptadas) ideas en materia de psicología, constituyeron la fórmula para la impresionante productividad de Eysenck. Solo o en colaboración con otros investigadores, dio forma a una obra en la cual podemos encontrar libros que pueden ser considerados como verdaderos clásicos de la psicología del siglo antecedente.

Errasti Pérez (1998) analiza de manera sucinta la obra escrita de este psicólogo:

"un primer repaso de esta obra sorprende por la variedad de sus contenidos, que abarcan desde pequeños libros divulgativos en paperback, -algunos tan populares como Usos y abusos de la Psicología-, hasta tratados académicos con títulos tan ambiciosos como La estructura de la personalidad humana o Estructura y medición de la Inteligencia (1979), desde publicaciones en donde se refinan complejas técnicas estadísticas como el análisis factorial o el análisis criterial hasta estudios acerca de la posibilidad de que los astros influyan de alguna manera sobre ciertas conductas humanas, pasando por trabajos sobre la eficacia de las psicoterapias, las actitudes sociales y políticas, la conducta sexual y marital, la relación entre el tabaquismo y el cáncer, los efectos de los suplementos de vitaminas sobre el cociente intelectual, los determinantes psíquicos de la criminalidad, y muchos otros" 
Usos y abusos de la psicología (Eysenck 1957), una obra en la cual "un psicólogo examina la validez de su ciencia en los asuntos humanos", es de las más leídas. Pero no solo ese libro: el Handbook of abnormal psychology, que él editara (Eysenck 1973), es una de las obras que -estamos seguros- será considerada como una de las más representativas del siglo XX.

Una de sus últimas obras, Genius: the natural history of creativity (Eysenck 1995), trata de un tema que ha despertado inmenso interés en la última década del siglo XX y en lo que va de la presente centuria, pero además propone una explicación audaz y desafiante.

Hemos mencionado solo algunos libros a guisa de ilustración, pero no podemos obviar una mención a Sexo y personalidad (Eysenck 1982b) así como a su excelente y aún bastante leído Fundamentos biológicos de la personalidad (Eysenck 1982a).

En todas estas obras se revela Eysenck como original científico y como aventajado escritor. No deja de sorprender su capacidad para desarrollar un estilo de comunicación a la altura del público especializado en unos libros, y al alcance del lector lego en otros.

Pero tal vez el rasgo de mayor fuerza es su actitud crítica, acerba, dura, por momentos casi sectaria, frente al psicoanálisis. Estamos tentados de emplear el término compulsión para referirnos a la frecuencia con la cual atacó al psicoanálisis, terminando por desecharlo como enfoque científico (véase Eysenck 1986).

Su radical postura le valió fama, pero también rechazo. No todos los que miran con escepticismo al psicoanálisis compartirán las siguientes afirmaciones suyas:

"Para mí, Freud era un cuentista, un novelista; un creador de mitos, si quiere, como Marx y toda esta gente: ha ejercido una tremenda influencia en las personas y en la sociedad, en la literatura, etc., pero pienso que ninguna de las teorías que propuso son defendibles ni, en rigor, científicas, ya que no pueden clasificarse; son demasiado vagas, demasiado generales. Desde mi punto de vista, fue realmente una gran desgracia para la evolución de la psicología y de la psiquiatría científica. Nos hizo retroceder unos cincuenta años" (Eysenck 1987, pg. 308).

¿Cuáles son las objeciones que formula a la teoría freudiana? Podríamos resumir todos los ataques que él lanza a esa doctrina en cuatro o cinco puntos. Uno es que el psicoanálisis es una construcción hipotética que clama validez universal pero que solo está basado en las experiencias del Freud clínico. Otro, que la suposición del complejo de Edipo y las atribuciones de causalidad para la neurosis no pasa de ser eso, una suposición, que no ha sido y muy probablemente no puede ser demostrada experimentalmente. Precisamente por ello el psicoanálisis no puede reclamar para sí la condición de ciencia, porque sus planteamientos central es no han sido verificados a través del trabajo experimental. Por si fuera poco, a la hora de interpretar hechos históricos, Freud y sus seguidores ignoran datos facticos y formulan interpretaciones personales sin fundamento en la práctica. Y por último, que como técnica psicoterapéutica no está demostrado que el psicoanálisis sea efectivo sino que es muy probable que inclusive dañe a las personas.

Eysenck apostó por un enfoque rotundamente objetivo en el estudio de la personalidad, sometiendo a dura crítica a las que no satisfacen estándares científicos. Errasti Pérez (1998) señala cinco 
características de su psicología: ver al hombre como un organismo biosocial, y a la mente y el cuerpo como un continuo solo diferenciado cuantitativamente, la reconciliación de los enfoques correlacional y experimental, la ausencia de división entre la psicología aplicada y la psicología básica, y la necesidad de fundamentar cualquier afirmación sobre bases empíricas.

Para él la personalidad puede ser entendida como una jerarquía conformada por respuestas específicas, respuestas habituales, rasgos y dimensiones. En la búsqueda de esas dimensiones identifica tres: extraversión-intraversión, estabilidad-inestabilidad y psicoticismo.

En el estudio de estas tres dimensiones Eysenck empleó cuestionarios e hizo uso extensivo del análisis factorial. Su famoso EPI (Eysenck Personality Inventory) debe ser uno de los reactivos psicométricos más conocidos y empleados.

Una personalidad rica, compleja y contrastante como la de Eysenck, no solo tenía que formular teorías sugestivas y libros reeditados y traducidos. También tenía que incursionar en temas controversiales, que terminarían convirtiéndolo en el blanco de múltiples ataques. Tal el caso de su posición con respecto a las relaciones entre herencia e inteligencia.

El tema no puede ser más controversial y explosivo y la literatura sobre el particular es abundante y de muy variada calidad. Por muchos años Cyril Burt defendió de modo rotundo la posición hereditaria sobre el particular. Eysenck se acogió a ella con su acostumbrada rotundidad. Por ese motivo no solo defendió a Burt sino también a Jensen.

Sin embargo, tras la muerte de Burt la obra de éste fue sometida a severo escrutinio y se llegó a la conclusión de que había cometido fraude (Hearnshaw 1979). Como consecuencia de esto, las críticas contra Eysenck arreciaron y llegaron al ataque personal y a la descalificación de sus planteamientos considerándolos como racistas.

Su concepción de la inteligencia, sin embargo, no solo estuvo influenciada por las ideas del discutido -y finalmente desacreditado- Burt. Jensen (1986) señala que "cuatro adjetivos caracterizan la posición de Eysenck en el estudio de la inteligencia: objetiva, cuantitativa, analítica y biológica" (pg. 89). En estos adjetivos se pone de manifiesto siempre según Jensen, también la influencia de Galton y Spearman, que junto con Burt, son los principales fundadores de la escuela inglesa de la psicología diferencial. Y deberíamos agregar a Donald Hebb (1904-1985), importante psicólogo canadiense.

Pero no fue solo el tema de la herencia el que puso a Eysenck en el centro de la polémica. Muchos años antes, ya en 1952, un artículo, "The effects of psychotherapy: an evaluation" (Eysenck 1952), en el cual ponía en tela de juicio la efectividad de la psicoterapia, le valió ataques y críticas no menos severos.

En ese trabajo, Eysenck presentaba la opinión de que la recuperación espontánea podía ser el real factor para que muchas personas se recuperaran o experimentaran alivio a sus problemas psicológicos. No es difícil imaginarse la recepción de estas ideas. 
Cedamos la palabra a esta altura a Angela Schorr (1982), que en su historia de la terapia conductual escribe:

"Que el psicoanálisis no era un tratamiento particularmente efectivo, lo sabía también la mayoría de los psicólogos clínicos. "Sin embargo", señala O. Hobart Mowrer, "vacilaban a renunciar a una teoría comprensible". No por último, un trabajo relativamente poco llamativo y de pocas páginas de Hans Jürgen Eysenck habría de escandalizar en 1952. Eysenck, que hacia fines de los años cuarenta se encontraba en los Estados Unidos, para informarse acerca de la nueva psicología clínica, no concordaba con sus colegas americanos que era justificado representar un enfoque insuficiente solo porque no había alternativas. Mientras ellos pensaban que se debía permanecer diplomático y tomar el desarrollo científico de modo paulatino, Eysenck se decidió por una estrategia de confrontación" (pg. 140).

Algo que concordaba perfectamente con el modo de ser de Eysenck, que en modo alguno eludía la confrontación y que, una vez involucrado en una polémica (y él fue el protagonista de muchas), expresaba de modo claro, rotundo, con un claro toque de agresividad, sus puntos de vista así como atacaba los de su antagonista. Y, por lo general, los temas que Eysenck abordaba eran tierra fértil para polémicas y controversias (la estructura de la personalidad, la determinación biológica de la inteligencia, las relaciones entre el tabaco y el cáncer, las diferencias de inteligencia y su relación con la raza), optando él por posiciones que despertaban el acalorado rechazo de muchos ${ }^{3}$.

En su Historia de la modificación de la conducta Kazdin (1983) resume con las siguientes palabras el significado de ese trabajo:

"Eysenck no encontró pruebas concluyentes de que la psicoterapia fuera más efectiva que la remisión espontánea (mejora que se produce sin tratamiento específico). La revisión se hizo famosa y extraordinariamente polémica debido a que ponía en tela de juicio la eficacia de la terapia tradicional. A raíz de ella empezó a surgir un interés cada vez mayor por la experimentación en la psicoterapia en general y por las limitaciones, o posibles limitaciones, de la terapia tradicional" (pg. 140).

Eysenck no fue un psicoterapeuta pero suele ser mencionado como uno de los pioneros de la terapia comportamental, precisamente debido a la crítica que él formula a las psicoterapias de corte psicoanalítico y a la pobre calidad de la investigación acerca de los reales efectos de la psicoterapia (Rachman 2016), así como a su insistencia en la necesidad de entender los trastornos del comportamiento a partir de una perspectiva fundamentalmente psicológica en la cual los hallazgos de la psicología del aprendizaje y de la teoría pavloviana se integran con hallazgos de la psicofisiología de la época y los resultantes de la aplicación del análisis factorial. En su empeño por combinar las más sólidas teorías y técnicas de la psicología experimental con los más precisos procedimientos de evaluación de las diferencias individuales (Revelle 2016), Eysenck sentó las bases teóricas para las terapias comportamentales.

3. Buchanan (2011) lo califica como "the most divisive figure British psychology has ever produced". 


\section{La difusión de las ideas de Frankl y Eysenck en el Perú}

Como sucedió con muchos teóricos europeos de su época, Frankl y Eysenck fueron dados a conocer entre nosotros gracias a la obra de Honorio Delgado (1892-1969), la más destacada personalidad de la psiquiatría peruana e hispanoparlante en el siglo XX.

Así es: en varios de sus trabajos Delgado menciona a Eysenck (si bien es verdad que lo hace de manera incidental), mientras que en su leído Curso de psiquiatría se refiere a la logoterapia en el rubro de la psicagogía.

La logoterapia es, en las palabras de Delgado, un

"método que consiste en llevar al foco de la conciencia las disposiciones espirituales subconscientes o descuidadas, a fin de hallar acicate en el mundo de los valores para reorientar la propia vida" (pg. 432).

Prosigue Delgado con una prosa que queremos transcribir:

"Es una aplicación práctica del filosofar sobre sí mismo, "análisis de la existencia" que apela a la fuente íntima de la libertad y la responsabilidad. Persigue la vivificación de lo posible íntimo, esclareciendo la significación trascendental del sufrimiento, del trabajo, del amor y de la existencia misma. El paciente, con su peculiaridad, con la estructura de su pasado, concentra su conciencia frente al cumplimiento por venir de sí mismo como empresa de salvación. El empeño del psicoterapeuta debe ser culminado hacia el logro de una vivencia radical de la responsabilidad en el enfermo. Los sentimientos religiosos constituyen elemento primario del análisis frankliano de la existencia, que postula el Dios subconciente" (pg. 432).

En los años setenta y ochenta Eysenck es en el Perú ya un nombre familiar para psicólogos y psiquiatras. José Anicama presentó una tesis de bachiller en la Universidad Nacional Mayor de San Marcos basada en sus ideas (Anicama 1974). Su libro Fundamentos biológicos de la personalidad (Eysenck 1982) goza en esos años de amplia lectoría y su muy conocido EPI (Inventario de la Personalidad de Eysenck) es utilizado en numerosas tesis y trabajos de investigación. El despliegue de recursos matemáticos que se puede observar en sus libros, y en especial el empleo de en esos años aún poco empleado análisis factorial, añaden elementos de prestigio a la inherente calidad científica de sus escritos.

No sucedía lo mismo en ese entonces con las ideas de Frankl, poco difundidas entre nosotros. Sus libros, publicados en versión castellana por el Fondo de Cultura Económica, de México, y la Editorial Herder, de Barcelona, eran poco conocidos y sus ideas no armonizaban con el entusiasmo que se experimentaba en aquellos años no solo en el Perú sino también en toda América Latina por los planteamientos y procedimientos de Skinner y del análisis de la conducta.

Ya en 1954 Frankl había visitado la Argentina y encontrado acogida para sus ideas, pero por mucho tiempo fue ese el único país latinoamericano en el cual se podía reconocer un claro interés por él. Treinta años más tarde, Frankl asistió al Primer Encuentro Latino-Americano HumanísticoExistencial: Logoterapia, en la Pontificia Universidad Católica de Rio Grande do Sul, en la ciudad de 
Porto Alegre. A estos dos viajes que constituyen el primer contacto del creador de la logoterapia con esta parte del mundo, debemos agregar otros (siempre a Argentina y a Brasil).

Eysenck, por su parte, también visitó en varias oportunidades la región latinoamericana. Flores Mendoza et al (2016) enumeran tres estancias suyas, en Argentina, México y Brasil.

\section{¿Cómo se recordará a uno y otro?}

Han pasado veinte años desde la desaparición física de ambos. A Eysenck se le sigue leyendo, sus obras se siguen reeditando y se las encuentra con facilidad en los anaqueles de las bibliotecas especializadas; las revistas fundadas por él (Behavior Research and Therapy, fundada en 1963, Personality and Individual Differences 1980) y que él dirigiera por varios años continúan apareciendo, todo lo cual es una expresión de la vitalidad y de la importancia de su obra ${ }^{4}$. Aun su artículo de 1952 es mencionado hoy con cierta asiduidad.

Un aspecto de esa vitalidad es la polémica que ella sigue generando. Para decirlo en dos palabras: solo se puede polemizar sobre algo que todavía es actual. Lo enterrado y lo olvidado no pueden dar lugar más que al recuerdo respetuoso o a la ritual referencia histórica.

Pero hay que reconocer que la frecuencia de sus citaciones ha disminuido. Y probablemente eso ha sucedido porque entre tanto se ha tomado distancia de la radicalidad de sus posiciones. Su objetivismo extremo no encaja, por decirlo de alguna manera, con el relativismo de nuestros días en el proceso de comprender los fenómenos psicológicos, derivado e influido notoriamente por el proceso de globalización. Sin embargo, los avances que provienen de las técnicas de neuroimagen y de la genética molecular, parecen dar la razón a Eysenck en lo que se refiere a sus planteamientos de la determinación biológica de la inteligencia (Gottfredson 2016).

En el caso de Frankl, su sistema psicoterapéutico ha logrado difundirse por el mundo entero y, en una época en la cual la desazón interna y el desencanto con la realidad de nuestros días se extienden, la logoterapia parece constituir un enfoque que posibilita la reflexión interna, la reconciliación con los valores que están en la potencialidad de cada uno de nosotros.

Abrami (2016) encuentra puntos de contacto entre las ideas de Frankl y el activo movimiento de la psicología positiva que, asimismo, despierta un interés cada vez mayor. Thir \& Batthyány (2016) han pasado revista hace poco al estado de la investigación empírica en torno a la logoterapia y de la lectura de lo que ellos señalan no se puede menos que concluir que las ideas y teorías de Frankl gozan de muy buena salud.

4. Es de interés revisar el número especial (volume 103, diciembre del 2016, "Hans Eysenck: One hundred years of psychology", pp. 1-220) de Personality and Individual Differences, dedicado Eysenck. 


\section{REFERENCIAS}

Abrami L.M. (2016) The Importance of meaning in Positive Psychology and Logotherapy. En: Batthyány A., ed., Logotherapy and Existential Analysis. Logotherapy and Existential Analysis: Proceedings of the Viktor Frankl Institute Vienna, vol 1., New York Springer, 303310.

Anicama, J. (1974). Rasgos básicos de personalidad de la población de Lima: Un enfoque experimental. Tesis de Bachiller en Psicología. Lima: Universidad Nacional Mayor de San Marcos.

Bettelheim, B. (1973). El corazón bien informado. La autonomía en la sociedad de masas. México DF: Fondo de Cultura Económica.

Buchanan, R. D. (2011). Looking back: the controversial Hans Eysenck. The Psychologist, 24, abril, 318-319.

Costa, P. T. \& McCrae, R. R. (1986). Major contributions to the psychology of personality. En: Mogdil, S. \& Mogdil, C., eds., Hans Eysenck. Consensus and controversy, Filadelfia - Londres, The Falmer Press, 63-71.

Danzer, G. (2002). Viktor Frankl - Psychotherapie als Suche nach Sinn. En: Levy, A. \& Mackenthun, G., eds., Gestalten um Alfred Adler. Pioniere der Individualpsychologie, Würzburg, Koenigshausen \& Neumann, 63-80.

Dunlap, K. (1928). A revision of the fundamental law of habit formation. Science, 67, 360-362.

Dunlap, K. (1932). Habits: their making and unmaking. New York: Liveright.

Errasti Pérez, J. (1998). Usos y abusos de la psicología de Eysenck. Psicothema, 10, 517-533.

Eysenck, H. J. (1947). Dimensions of personality. Londres: Routledge \& Kegan Paul.

Eysenck, H J. (1952). The effects of psychotherapy: an evaluation. Journal of Consulting and Clinical Psychology, 16, 319-24.

Eysenck, H. J. (1957). Usos y abusos de la psicología. Madrid: Biblioteca Nueva.

Eysenck, H. J., ed. (1973). Handbook of abnormal psychology. San Diego: Robert R. Knapp.

Eysenck, H. J. (1982a). Fundamentos biológicos de la personalidad. Barcelona: Fontanella.

Eysenck, H. J. (1982b). Sexo y personalidad. Madrid: Cátedra.

Eysenck, H. J. \& Nias, D. K. B. (1982). Astrology. Science or superstition? Harmondsworth, Middlesex: Penguin.

Eysenck, H. J. (1986). Decline and fall of the Freudian Empire. Harmondsworth: Penguin Books 
Eysenck, H. J. (1987). Entrevista. En: Evans, R. I, comp., Los artífices de la psicología y el psicoanálisis. Conversaciones con los grandes psicólogos contemporáneos, México, D. F., Fondo de Cultura Económica, 301-316.

Eysenck, H. (1995) Genius: The Natural History of Creativity. Cambridge: Cambridge University Press.

Eysenck, H. J. (1998). Intelligence: A New Look. New Brunswick, NJ: Transaction Publishers.

Fizzotti, E. (1977). De Freud a Frankl. Interrogantes sobre el vacío existencial. Pamplona: EUNSA.

Flores-Mendoza, C.; Ardila, R.; Gallegos, M.; Sampaio Braga, B. \& Marcos Andrade, D. (2016). Hans Eysenck in Latin America: his influence in the psychology, the study of personality, and individual differences. Personality and Individual Differences, 103, 68-73.

Frankl, V. E. (1938). Zur geistigen Problematik der Psychotherapie. Zentralblatt für Psychotherapie, $10,33-45$.

Frankl, V. E. (1964). Teoría y terapia de las neurosis. Madrid: Gredos.

Frankl, V. E. (1995). Was nicht in meinen Büchern steht. Munich: Quintessenz.

Frankl, V. E. (1970). Psicoanálisis y existencialismo. México, D.F.: Fondo de Cultura Económica.

Frankl. V. E. (1979). La presencia ignorada de Dios. Barcelona: Herder.

Frankl, V. E. (1991). El hombre en busca de sentido. Barcelona: Herder.

Frankl, V. E. (1994). Logotherapie und Existenzanalyse. Texte aus sechs Jahrzehnten. Munich: Quintessenz.

Goldberg, D. ; Blackwell, B. \& Taylor, D. (2015). Professor Sir Aubrey Lewis, The Maudsley Hospital \& The Institute of Psychiatry. International Network for the History of Neuropsychopharmacology; http://inhn.org/biographies/sir-aubrey-lewis-bydavid-goldberg-barry-blackwell-and-david-taylor.html; recuperado el 24 de junio del 2017.

Gudjonsson, G. H. (1997). Hans Jürgen Eysenck. Emeritus Professor of Psychology, Institute of Psychiatry, University of London. Born 4 March 1916; died 4 September 1997 (aged 81). Personality and lindividual Differences, 23, 711- 712.

Gottfredson, L. S. (2016). Hans Eysenck's theory of intelligence, and what it reveals about him. Personality and Individual Differences, 103, 116-127.

Hearnshaw, L. S. (1979). Cyril Burt, psychologist. Londres: Hodder and Stoughton.

Hobsbawn, E. (1995). Historia del siglo XX. Barcelona: Crítica.

Jensen, A. R. (1986). The theory of intelligence. En: Mogdil, S. \& Mogdil, C., eds., Hans Eysenck. Consensus and controversy, Filadelfia - Londres, The Falmer Press, 89-102. 
Kagan, D. (2003). Sobre las causas de la guerra y la preservación de la paz. Madrid - México, D. F.: Turner - Fondo de Cultura Económica.

Kazdin, A. E. (1983). Historia de la modificación de conducta. Bilbao: Desclée de Brouwer.

Lukas, E. (1985). Auf der Suche nach Sinn. Logotherapie. En: Petzold, H., de., Wege zum Menschen. Methoden und Persönlichkeiten moderner Psychotherapie, Ein Handbuch (vol. 1), Paderborn, Junfermann-Verlag, 451-522.

Pongratz, L. J. (1975). Lehrbuch der Klinischen Psychologie. Psychologische Grundlagen der Psychotherapie. Gottinga: Verlag für Psychologie - Dr. C. J. Hogrefe.

Rachman, S. J. (2016). Hans Eysenck's contributions to clinical psychology and behavior therapy. Personality and Individual Differences, 103, 91-92.

Rattner, J. (1990). Klassiker der Tiefenpsychologie. Munich: Psychologie Verlags Union.

Revelle, W. (2016). Hans Eysenck: personality theorist. Personality and Individual Differences, 103, 32-39.

Riedl, J. (1995). Viena infame y genial. Madrid: Anaya \& Mario Muchnik.

Rushton, J. P. (2001). A scientometric appreciation of H. J. Eysenck's contributions to psychology. Personality and Individual Differences, 31, 17-39.

Schorr, A. (1984). Die Verhaltenstherapie. Ihre Geschichte von den Anfängen bis zur Gegenwart. Weinheim - Basilea: Beltz.

Sheperd, M. (1980). From social medicine to social psychiatry: the achievement of Sir A u b r e y Lewis. Psychological Medicine, 10, 211-218.

Spiegelberg, H. (1985). Die Rolle der Phänomenologie in Viktor Frankls Logotherapie und Existenzanalyse. En: Längle, A., ed., Wege zum Sinn. Logotherapie als Orientierungshilfe, Munich, Piper, 55-70.

Thir, M. \& Batthyány, A. (2016). The state of empirical research on logotherapy and existential analysis. En: Batthyány, A., ed., Logotherapy and existential analysis. Logotherapy and existential analysis: Proceedings of the Viktor Frankl Institute Viena 1, .Springer International Publishing, 53-74.

Weinzierl, U. (1997). Leiden heisst auch reifen. Zum Tod des österreichischen Psychotherapeuten und Kulturkritikers Viktor E. Frankl. Frankfurter Allgemeine Zeitung, edición del 4 de setiembre, pg. 35. 